\title{
Infrared Method for Determining Circadian Patterns of Carbon Dioxide Release*
}

\author{
W. C. Levengood \\ Institute of Science and Technology, The University of Michigan Ann Arbor, \\ Michigan 48107
}

Received January 7, 1969

\begin{abstract}
Summary. Using an infrared technique based on the continuous monitoring of a characteristic infrared absorption band, temporal variations in the circadian patterns of carbon dioxide release were examined in two species of noctuid moths, the corn earworm Heliothis zea (BODDIE) and the fall armyworm Spodoptera frugiperda (J. E. Sиттн). The detailed emission patterns were shown to be different in the two moth species (Figs. 3, 4). Emission patterns similar to those obtained in adults were also observed to be present in the pupal stage of development.

The influence of light-dark conditions was examined and in the pupal stage the mean rate of carbon dioxide evolution was lower in those pupae exposed to a dark chamber compared with pupae of corresponding age and sex exposed to approximately 80 foot candles of light. This light-dark effect was not observed in adult moths. An insect attractant lamp emitting radiation in the blue and ultraviolet regions of the spectrum was found to have a pronounced stimulating influence. Over a 24 hour period of observation, the carbon dioxide buildup was approximately two times greater from male $S$. frugiperda moths exposed to the attractant lamp when compared with control moths exposed to room light or dark chamber conditions (Fig. 5). Alternate 24 hour exposures to the attractant lamp and dark conditions disclosed that the moths returned to a low level of emission following each light exposure. The attractant lamp also altered the phasing or onset of the circadian pattern of carbon dioxide release, however, the sequential patterns of release remained the same.
\end{abstract}

\section{Introduction}

A new technique is presented for determining the characteristic patterns of carbon dioxide emitted as an end product in metabolism. The method is based on the measurement of a characteristic infrared absorption band located at 4.3 microns and detected in the gas exchange process within closed systems. This monitoring system was utilized to explore temporal variations in circadian carbon dioxide release in two species of nocturnal moths, the corn earworm, Heliothis zea (BoDDIE) and the fall armyworm, Spodoptera frugiperda (J. E. SMITH). Details in

* This work was sponsored by the U.S. Department of Agriculture, Agricultural Engineering and Entomology Research Divisions, ARS, Beltsville, Md. Contract No. $12-14-100-8942(42)$. 
the patterns of emission are shown to be different in these two species; however, within each species, the general pattern of release is reproducible.

The occurrence of a daily maximum in the carbon dioxide release pattern preceded the nocturnal interval of flight activity. The rates of carbon dioxide release are presented for both pupae and adult stages and comparisons made between the emission from and males and females. In addition, with a dual chamber system the influence of external environmental factors such as light-dark photoperiodic conditions was examined. An ultraviolet attractant lamp known to be effective under field conditions was found to have a pronounced stimulating effect on the circadian pattern of carbon dioxide release.

Carbon dioxide release patterns have been intensively investigated in a number of insect species. The giant silkworm moth, Hyalophora cecropia, exhibits a cyclical release of carbon dioxide resulting in sharp bursts (Levy and SchNEIDERMaN, 1966) whereas other insects display more continuous rates of carbon dioxide emission (SLama, 1960). Most of the studies reported in the literature have, in most cases, been directed toward understanding the release patterns in relation to physiological alterations in the organism at various stages of development and, in some cases, under normal conditions. It appears, however, that there have been few concerted efforts to examine the diurnal patterns of carbon dioxide release in terms of temporal consistency. From the exploratory findings based on this infrared method it is very conceivable that such studies could shed light on the question of the normal release pattern being involved as an endogenous factor in the circadian rhythm. The overt behavior of nocturnal insects, as for example, mating responses or attractiveness to light traps, might be more clearly elucidated by gaining knowledge of normal periodic respiration and externally introduced factors which tend to alter this pattern.

\section{Experimental Methods}

The experimental technique is a modification of an optical method devised for monitoring the $\mathrm{pCO}_{2}$ level in physiological liquids (LEVENGOOD and WOLFE, 1964). Two identical chambers and gas cells are employed, the advantage being that the organism may be subjected simultaneously to a "control" and a "test" environment. Specific types of environmental parameters may be examined during the same time interval.

Each sealed system employed for collecting the carbon dioxide released by the moths, as shown diagramatically in Fig. 1, consists essentially of two interconnecting chambers. One chamber designated the "moth chamber" contains the moths or pupae. The second container, attached by means of interconnecting hose to the specimen chamber, serves as the "test cell" in which the carbon dioxide concentration is spectrophotometrically determined. The total volume of each 


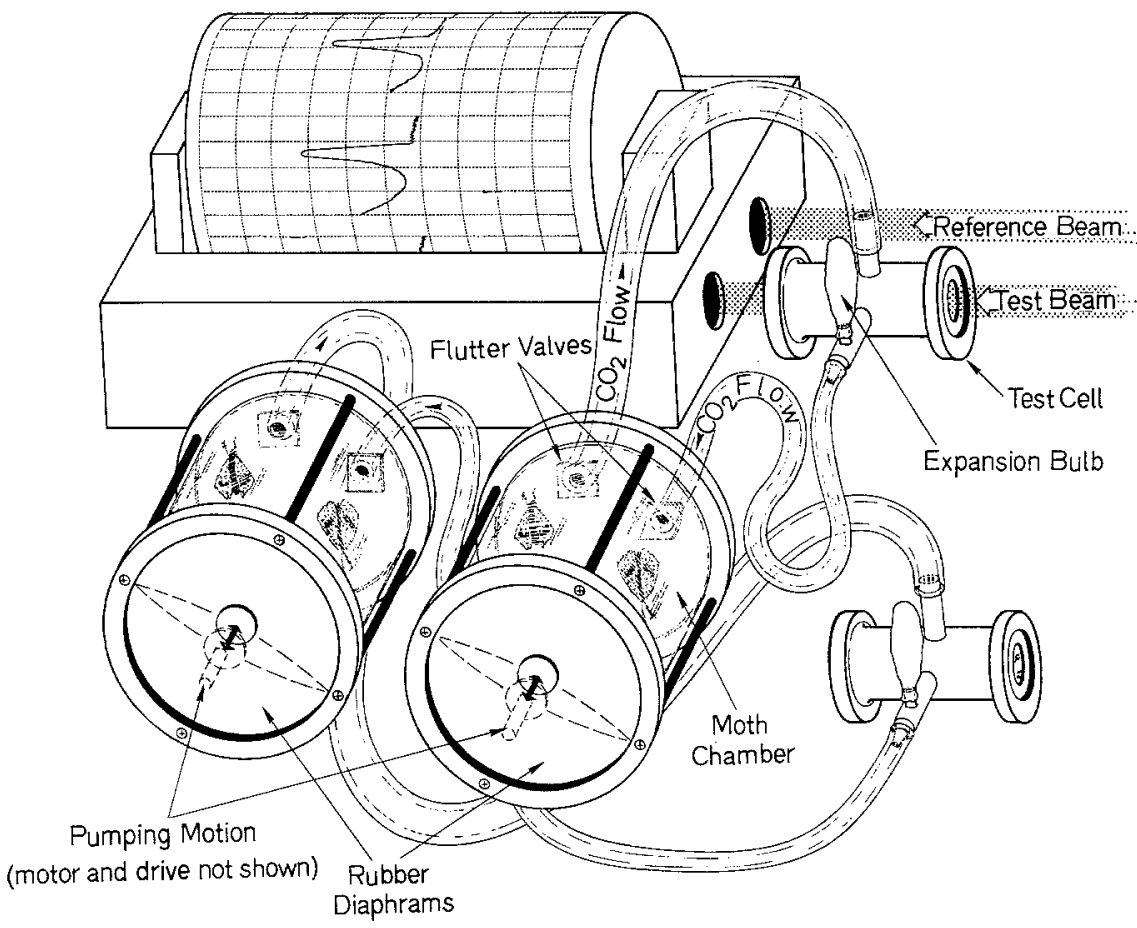

Fig. 1. Respiration chambers and attached gas cells mounted in front of the infrared spectrophotometer. One gas collecting cell in measuring position.

closed system is approximately $1100 \mathrm{~cm}^{3}$ including the specimen chamber interconnecting hose and gas collecting cells. Each of the interconnecting pairs of chambers contains an internal air-circulation system to insure more rapid mixing of the emitted carbon dioxide than would take place by diffusion alone.

The dual chamber apparatus shown in Fig. 1 is mounted in front of a model 21 Perkin-Elmer ${ }^{1}$ infrared recording spectrophotometer used in the continuous monitoring tests of the carbon dioxide release patterns. One gas collecting test cell attached by hose to the specimen chamber on the right in Fig. $I$ is in position within the beam of the spectrophotometer. For each given measurement of concentration in the gas cell, a spectrum was traced from 3.8 to 5 microns. The wavelength of interest is at 4.3 microns, one of the fundamental absorption bands of carbon dioxide. This particular wavelength was utilized since there is very little attenuation due to water vapor within this spectral region.

The cylindrical portions of the moth chamber are composed of sections cut from plastic cylinders approximately $12 \mathrm{~cm}$ in diameter and $8 \mathrm{~cm}$ in length. Aluminum end plates are sealed to each plastic cylinder by means of 0 -rings and tightened down with retainer bolts. Inserted between the aluminum ring plates and the plastic cylinders are rubber diaphragms attached to a cam on a telecron

1. Mention of companies or products in this paper does not imply recommendation or endorsement by the U.S. Department of Agriculture over others not mentioned. 
motor. These rubber diaphragms were given approximately a $1 \mathrm{~cm}$ thrust at a rate of 50 cycles per minute, thus providing continuous air circulation in the closed system. The opposite aluminum end plates on the moth chambers contained openings for the inlet and exit tubes. Over these openings were mounted rubber "flutter valves" to provide more efficient gas circulation.

At the opposite end of $55 \mathrm{~cm}$ long interconnecting tygon hoses, were attached the gas collecting test cells fabricated from brass cylinders. At each end of the gas cells were face plates with machined $O$-rings to provide a gas tight seal for the window materials in the absorption cells. The windows are approximately $3.5 \mathrm{~cm}$ in diameter with an optical path length of $10 \mathrm{~cm}$. Initially a $0.01 \mathrm{~cm}$ thick polyethylene film was used for the windows in the gas collecting cells. Later the plastic was replaced by more rigid windows consisting of $0.32 \mathrm{~cm}$ thick sapphire. The sapphire also provided partial transparency in the 4.3 micron region. Inserted in one hose attached to each of the gas collecting test cells is a " $T$ " tube and over this is placed a latex diaphragm (pharmaceutical finger cot). This diaphragm serves as an "expansion bulb" to enhance the pumping action through the system. Using this internal air circulation technique, the response time after inserting a small amount of carbon dioxide in the test chamber was determined to be less than six minutes (time to reach equilibrium concentration).

A typical test was carried out by placing the desired number of moths (or pupae) in the chamber and then sealing after flushing the entire system with air. The flushing with dry air was to insure that the initial concentration of carbon dioxide was at ambient level. A series of measurements was generally initiated between 8:00 and 9:00 A. M., and a spectral trace of both cells was made at the start of each test. After these initial recordings, the spectra were alternately taken at half hour intervals. These alternate half hour measurements were generally continued for either 12 or 24 hour periods, depending on the type of test being conducted. Recently tests have been carried on continuously for $72 \mathrm{hrs}$ or more. For long period experiments, it is necessary to periodically flush the systems when the absorption band becomes pronounced. This flushing only interrupts the test for a few minutes and the monitoring is continued following this brief interruption. The ambient temperature during a test period was maintained at $25^{\circ} \mathrm{C}$ with a $\pm 0.5^{\circ} \mathrm{C}$ total variation. After a given test, the gas collecting cells were removed from the specimen chambers, thus allowing air to enter the chambers with normal exchange during the interval between the test runs. In the case of adult moths, a small plastic vial of sugar water was inserted in each chamber to provide food during the test period.

The gas collecting cells were calibrated by comparing the depth of the $4.3 \mu$ spectral band with known amounts of carbon dioxide inserted in the cells. By determining the depths of the absorption band and knowing the number of specimens in the test chamber, one may then very simply calculate the amount of carbon dioxide emitted per organism and for any given period of observation. Considering instrument and calibration variations, the rate of release was determined with an error of $\pm 0.02 \mathrm{ml} \mathrm{CO} /$ organism $/ \mathrm{hr}$.

In the following sections discussing the effects of various exogenous environmental parameters, the tests were conducted so that alternate chambers were exposed to the specific stimulus under investigation. That is, if the right chamber was exposed to a particular type of environment for a $24 \mathrm{hr}$ period, then on the next $24 \mathrm{hr}$ interval the left chamber was exposed to the same conditions with the right chamber now serving as the control. This method of alternate testing eliminated bias in terms of slight differences in the weights or activity of the specimens as well as variations in the apparatus. 

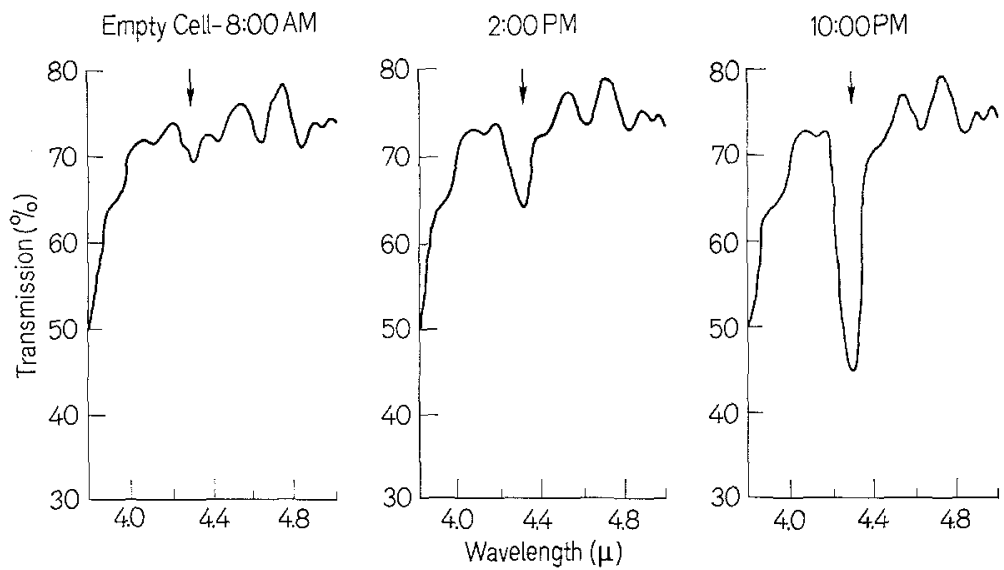

Fig. 2. Typical spectral traces showing buildup of the $4.3 \mu$ band (male $S$. frugiperda moths in chamber)

The two species of moths used in this study were raised on artificial diet. The basic formula for this diet was presented by Adkisson, VANDERzant, BULL, and ALLISon (1960) with specific details furnished by Mr. RoBERT Burton at the U.S. Department of Agriculture Laboratories, Tifton, Georgia. Since circadian patterns were examined in this study, it should be emphasized that during the rearing of these moths, there was no contact with outside lighting conditions. The moths were reared for successive generations under constant low illumination (less than 10 foot candles of light) and the respiration monitoring was conducted in an inside laboratory where only artificial lighting was used (approximately 80 foot candles). With adult moths two to six individuals were placed in the chambers (depending on which species was being tested). In the case of $H$. zea two moths were used; with an average weight of 0.2 grams this gives a sensitivity of approximately 0.1 grams adult tissue to produce a response.

\section{Results}

An example of the type of spectrometer traces obtained at three different time intervals is presented in Fig. 2 to illustrate typical data. These curves were obtained with adult, male $S$. frugiperda moths in the test chamber. The left curve shows the location of the $4.3 \mu$ band (indicated by the arrow) in the initial empty cell (ambient air) and the succeeding spectra demonstrate the increase in the depth of the absorption band with continued buildup of carbon dioxide.

During the test periods, the illumination consisted of fluorescent lighting (approximately 80 foot candles at the specimen chambers). In initial exploratory experiments, the chambers were covered at $7: 30$ P.M. with a black cloth until 8:00 A. M. the following morning. This was done to simulate day-night conditions; however, it was found that this 
7:30 darkening did not have any noticeable effect on the respiration rates, and was discontinued. A light-dark effect was discovered by covering the chambers earlier in the day and this will be more thoroughly discussed at a later point.

\section{Comparison of Species}

The carbon dioxide release over 24 hour intervals exhibit characteristically different patterns in the $H$.zea and S. frugiperda species. The rate curves in Fig. 3 were obtained from both the adult and pupal stages of $S$. frugiperda moths. The data are presented as milliliters carbon dioxide per moth (or pupa) per given hour of observation; it should be noted that different ordinate scales are used in Fig. 3 since the rates are much higher in the adult moths. The local (Eastern Standard) time is used throughout this study.

Considering first the adult moths, it may be seen that the primary maximum in both the male and female respiration curves occur between 5:00 and 6:00 P. M. with a second less pronounced maximum in the morning hours (10:00-11:00 A.M.). The primary maximum in the adult respiration curves occurs at a period just preceding the observed nocturnal interval of flight activity (8:00 P.M.—2:00 A.M.). This disappearance of $\mathrm{CO}_{2}$ emission with activity is in accord with the findings of others. Sharp emission peaks of emitted $\mathrm{CO}_{2}$ have been reported to disappear when insects become active (MrLLER, 1964). As shown in a later section, the onset time of the high emission peaks may vary somewhat; however, the shape of the emission curve remains essentially unchanged from one generation to the next.

It is interesting to note that the six to seven day old pupae in Fig. 3 also disclose the pronounced maximum in the respiration, and there is also an indication of the lesser secondary maximum preceding the rapid rise.

The basic carbon dioxide release characteristics of the corn earworm moth, Heliothis zea, are presented in Fig. 4. A general comparison of this species with the fall armyworm data presented in Fig. 3 demonstrates that the respiration curves are different in detail. The respiration rates are much higher in the $H . z e a$ moth, however, the primary maximum occurs in the early evening hours as in the case of the fall armyworm. The drop-off after the maximum is not, however, as clearly defined in Fig. 4 and the rate remains fairly high until after midnight. The first peak in the $H . z e a$ rate curves occurs at around 1:00 P.M. which is approximately two hours later than found in the fall armyworm.

The respiration rate curves for the H. zea pupae although of lower magnitude are similar to the adult curves with the maximum occurring around 6:00 to 7:00 P.M. again with the less rapid decrease following 


\section{S. frugiperda}
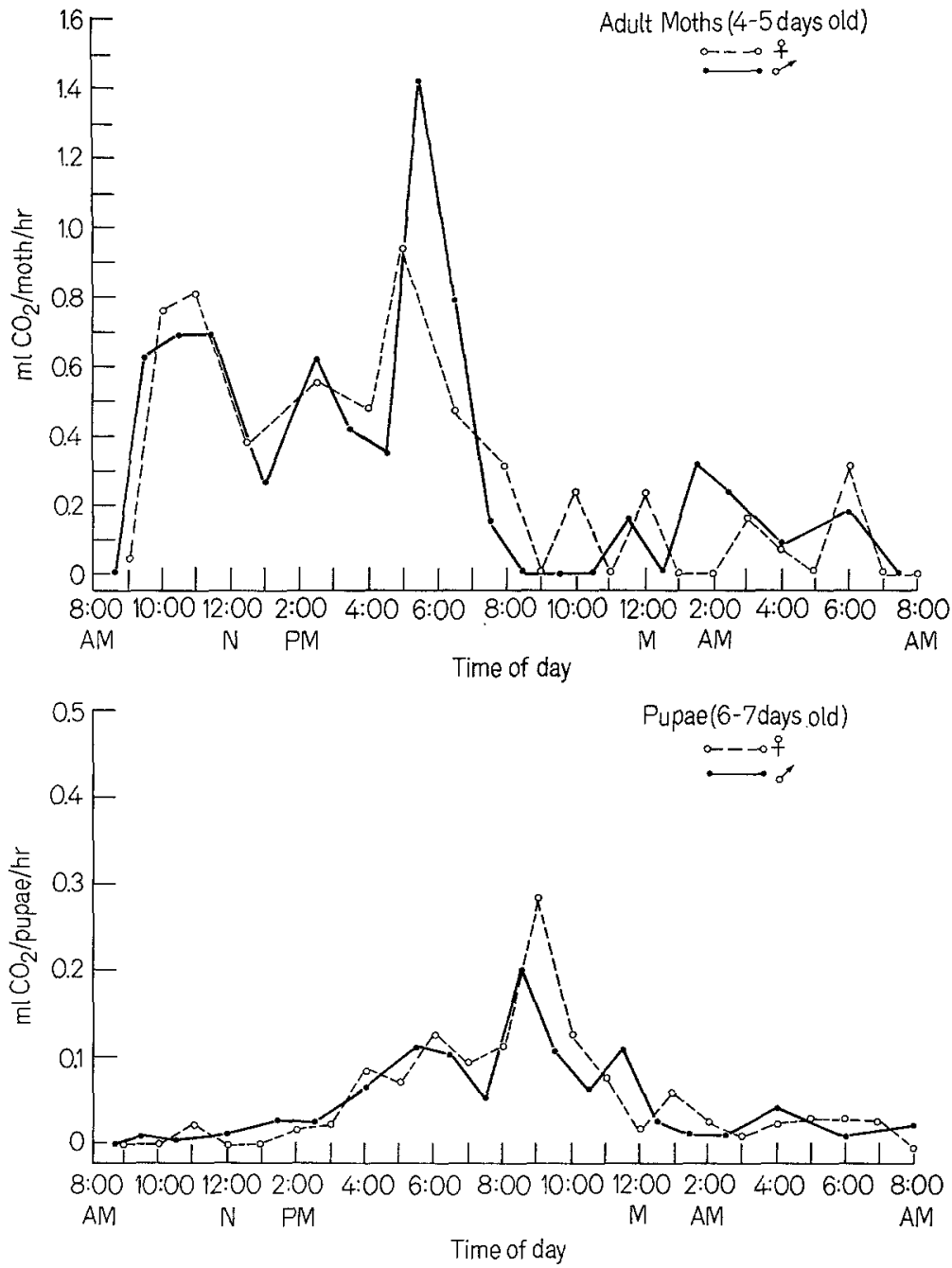

Fig. 3. Carbon dioxide rates for adult and pupal stages of $S$. frugiperda moths

the maximum rate. The $H$. zea pupae clearly demonstrate different respiration rate curves than produced with the fall armyworm pupae; however, the six to seven day old pupae in Fig. 4 are not at the same corresponding age in the pupal cycle as the six to seven day old fall armyworm pupae. The period of pupal development for the H. zea is approximately 16 days, whereas for the $S$. frugiperda the period is only around 10 days. 

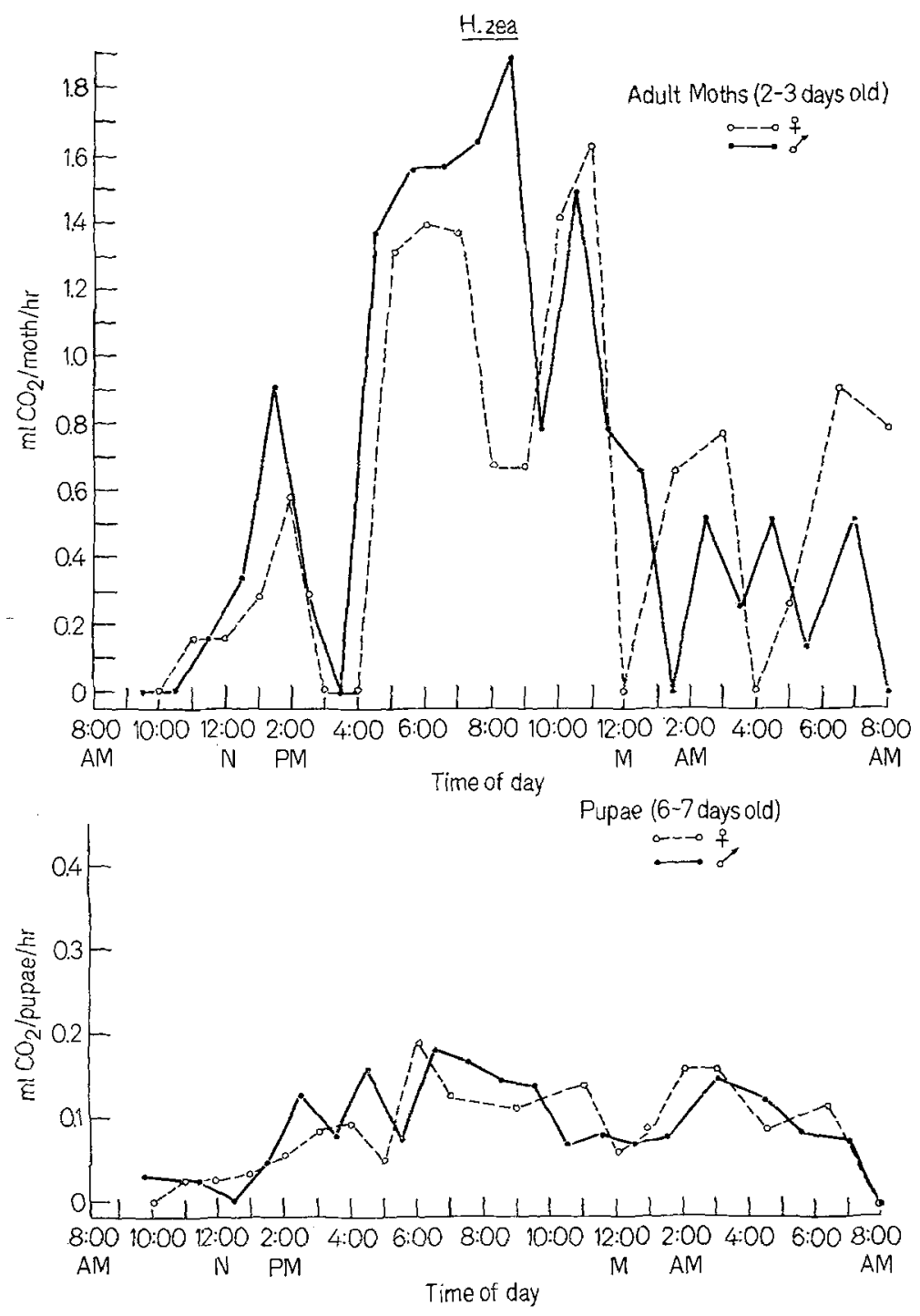

Fig. 4. Carbon dioxide rates for aduit and pupal stages of $H$. zea moths

Viability and Sex Difference

To examine the possibility that the respiration test may influence the viability of the organism, unmated $H$. zea moths were removed from the respiration apparatus and placed in rearing containers (battery jars) to examine viability. After four days, eggs were seen and larvae hatched. This demonstrates that the respiration tests do not interfere 
with the general viability of the organism. In recent studies, it has been found that the adult moths will mate within the respiration chambers and deposit viable eggs. During a $24 \mathrm{hr}$ test period the total buildup of carbon dioxide in the specimen chambers generally does not exceed a $1 \%$ concentration.

Although the moths (or pupae) were separated according to sex and placed in separate specimen chambers, the respiration rates for both the males and females are quite similar in appearance. One cannot point to any pronounced clear cut differences between the respiration rates of the males and females; however, it might be said that in the case of the adult moths, the males in general show a slightly higher rate of respiration, particularly at the period of the primary maximum respiration. It is noteworthy that even though the male and female groups were in chambers completely isolated from one another, the rate curves are remarkably similar in detail; the secondary and primary peaks of emission occur roughly within the same temporal intervals. From the results with the pupae, it appears that these circadian respiration patterns are, to a certain degree, established before the organism reaches the adult stage.

\section{Influence of Light-Dark Conditions}

The emission patterns presented in Figs. 3 and 4 were obtained by continuously illuminating the specimen chambers with white fluorescent lights ( 80 foot candles) during the entire 24 hour period of observation. The influence of light-dark conditions was examined in the following manner. First, the same number, age, and sex, of moths or pupae were placed in each of the specimen chambers. After sealing the system, one of the chambers was covered and completely darkened (on consecutive tests, the left and right chambers were alternately darkened to insure no positional bias). A given test run was started around 8:00 A.M. with the nondarkened chamber exposed to the laboratory white light illumination (ambient temperature the same in both chambers). These tests were conducted during a twelve hour interval, that is from 8:00 in the morning until 8:00 in the evening.

The light-dark conditions were initially investigated using pupae of $H$. zea with five pairs of young pupae placed in each chamber and the respiration patterns examined as a function of pupal age. From the hourly spectral curves an average value of the rate was obtained over the 12 hour period of examination for both the light and dark exposed chambers. These values are listed in Table 1 , and it is clear that the mean rate of carbon dioxide evolution is consistently lower in those pupae exposed to the dark chamber during the $12 \mathrm{hr}$ test interval. The differences between the mean emission values for the light and dark 
exposed chambers are not consistently the same over the cycle of pupal development. As shown in Table 1, the difference between the light and dark chamber is less pronounced in the early developmental stages than just before eclosion. The light-dark effect is less pronounced when the

Table 1. The influence of light and darkness on the respiration rate in developing Heliothis zea pupae. The values were determined in $12 \mathrm{hr}$ intervals $18: 00 \mathrm{~A} . \mathrm{M}$. to 8:00 P.M.)

\begin{tabular}{lll}
\hline $\begin{array}{l}\text { Approximate } \\
\text { pupal age } \\
\text { (hr) }\end{array}$ & $\begin{array}{l}\text { Chamber illuminated } \\
\text { with } 80 \mathrm{ft}-\mathrm{c} \\
\left(\mathrm{ml} \text { of } \mathrm{CO}_{2} / \text { pupae} / \mathrm{hr}\right)\end{array}$ & $\begin{array}{l}\text { Darkened } \\
\text { chamber } \\
\left(\mathrm{ml} \text { of } \mathrm{CO}_{2} / \text { pupae } / \mathrm{hr}\right)\end{array}$ \\
\hline 80 & 0.117 & 0.086 \\
130 & 0.160 & 0.157 \\
245 & 0.140 & 0.127 \\
300 & 0.291 & 0.224 \\
360 & 0.319 & 0.231 \\
\hline
\end{tabular}

mean rate of carbon dioxide evolution is low or at the middle portion of pupal development. In general, it appears that the characteristics of the carbon dioxide emission follows the U-shaped pattern observed in other insect species (AGRELL, 1964).

An exposure of adult $H$. zea male moths to the same L-D regime produced no significant alteration in the emission patterns. Data demonstrating this lack of a light-dark effect in adult moths will be incorporated in results presented in the following section.

\section{Exposure to an Insect Attractant Lamp}

The response of noctuid moths to light sources emitting radiation in the blue and ultraviolet regions is well documented in the literature. There appears to be little information, however, pertaining to the possible influence of insect attractant lamps on physiological patterns. Field studies using light traps have shown that a commercial (GE) black light lamp designated "BL" is effective as an attractant (DEAY, BarretT, and HARTSOok, 1965). This lamp emits radiation in the blue-green region of the visible (mercury lines) with an additional component of ultraviolet emission.

A series of tests was conducted to determine the effect of these BL attractant lamps on the respiration patterns in adult moths. In these tests one of the respiration chambers was illuminated and the second chamber darkened for the entire 24 hour period of observation as described in the preceding section. A four watt BL lamp was mounted about $6^{\prime \prime}$ above the respiration cell and ventilation was provided to prevent heating within the specimen chamber. At the time 
these experiments were initiated, a supply of $\$$. frugiperda adult moths was available which was utilized in these experiments. Both the dark conditions and the BL exposure were continued for a 24 hour period of observation. The room lights above the spectrometer were turned off during this test so that the only illumination was from the BL at. tractant lamp.

In presenting data from these exposure tests, a somewhat different method of plotting was utilized, than discussed in the previous sections. Instead of presenting respiration rate curves and showing the spikes as they occur over the periods of observation, the rate curves were essentially integrated and the accumulated carbon dioxide plotted as a function of the diurnal cycle. Although this does not illustrate the pronounced rate alterations in such an obvious manner, it is more advantageous for demonstrating buildup and comparing total outputs of carbon dioxide. Steep rises in these accumulation curves represent high peaks in the rate curves such as those shown in Figs. 3 and 4.

The stimulating effect of the BL attractant lamp on moths exposed during the 24 hour period is demonstrated in the data summarized in Fig. 5 (taken during a $48 \mathrm{hr}$ test period). As mentioned in the preceding section, the lower curves in Fig. 5 demonstrate that in the case of adult moths there is no significant difference in the respiration patterns obtained in the dark chambers and under the constant illumination of fluorescent room lights. The BL attractant lamp on the other hand produces a low respiration rate in the early morning hours with a very pronounced rise at around midnight. The final accumulated carbon dioxide is approximately two times greater per moth in the case of the BL attractant lamp than under either the conditions of the room light or the darkened chamber.

For the purpose of examining a possible residual effect of the BL attractant lamps on the response patterns, the accumulated carbon dioxide was recorded on four consecutive days from one group of moths and the chamber was alternately exposed to the BL source and dark conditions. This sequence of data is presented in Table 2 and the total accumulated carbon dioxide for the 24 hour period is listed for each of

Table 2. Accumulated carbon dioxide from male S. frugiperda moths alternately exposed to BL attractant lamp and dark conditions (moths 3 days old at start)

\begin{tabular}{llr}
\hline Day & $\begin{array}{l}\text { Conditions } \\
\text { in moth chamber }\end{array}$ & $\begin{array}{l}24 \mathrm{hr} \text { accumulation } \\
\text { of } \mathrm{CO}_{2}(\mathrm{ml} / \text { moth })\end{array}$ \\
\hline 1 & BL Lamp & 13.9 \\
2 & Dark & 5.1 \\
3 & BL Lamp & 12.1 \\
4 & Dark & 1.0 \\
\hline
\end{tabular}




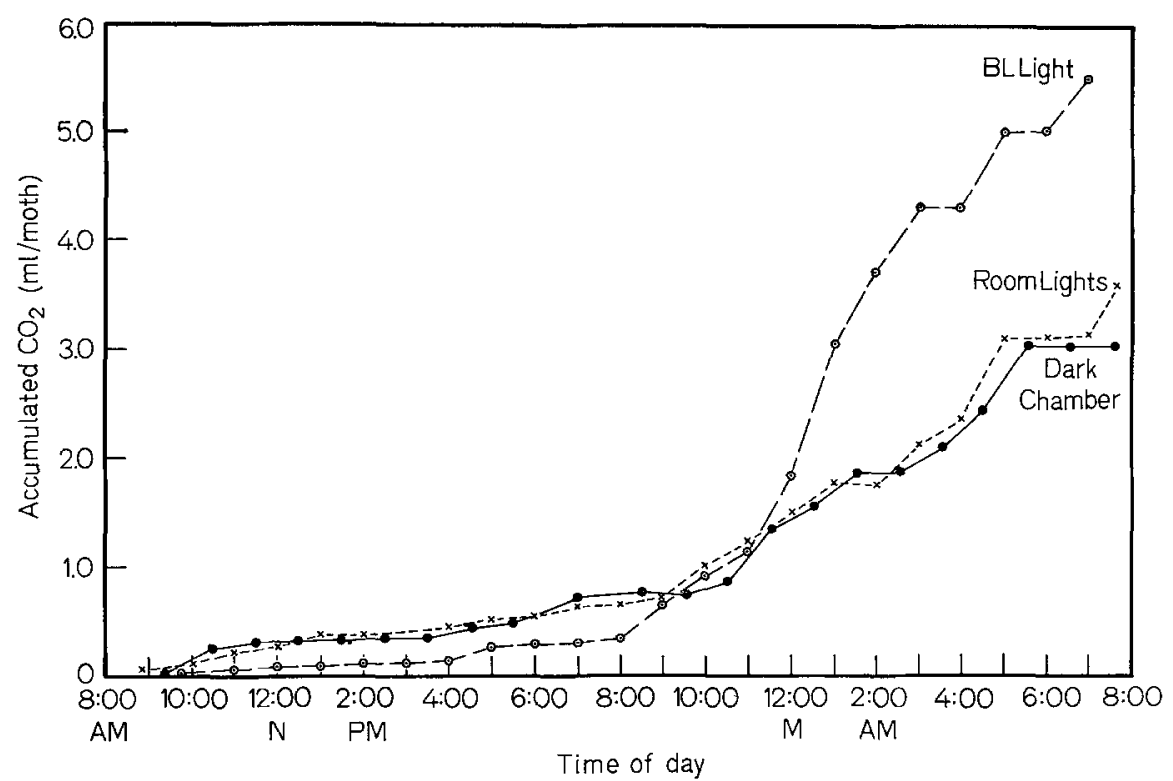

Fig. 5. Influence of BL insect attractant lamp on respiration patterns of male S. frugiperda moths (all data obtained in a $48 \mathrm{hr}$ period)

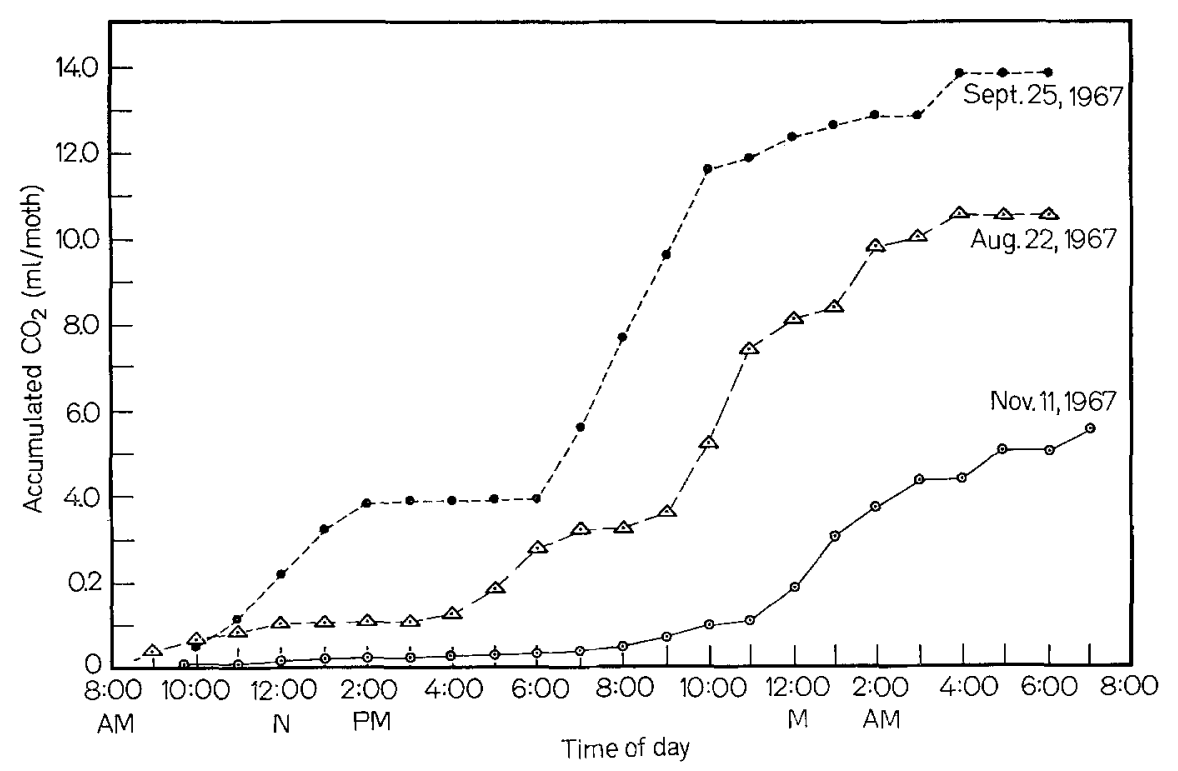

Fig. 6. Circadian accumulation of carbon dioxide in three different generation of laboratory reared male S. frugiperdo moths exposed to a BL attractant lamp (moths tested five days after eelosion) 
the environmental conditions. These data demonstrate that exposure to the BL lamp triggers the high emission rates whereas on alternate days of dark exposure the low level is again obtained. The very low accumulated carbon dioxide on the fourth test day may reflect an advanced senescence in these moths brought about by the stimulating action of the two previous BL exposures. On the fourth day of the test, these moths were chronologically seven days old, and several of the moths in the test chambers had expired, whereas under normal conditions, these moths generally live from 12 to 14 days. More tests of this nature will have to be conducted, however, before it can be stated with certainty that the high carbon dioxide emission stimulated by the BL attractant lamps was the responsible factor in shortening the lives of the moths used in this test.

It was also observed that the BL attractant lamps caused pronounced shifts in the onset time of the rapid rises or peaks in the rates of carbon dioxide emission. This influence was quite different for different generations of the moths as the data in Fig. 6 suggest. The data for the three generations presented in Fig. 6 were obtained from adult male $S$. frugiperda moths five days after eclosion. The striking feature of Fig. 6 is the fact that even though the rise times or the rapid rates occur at different times of the day, all three curves are very similar in appearance. The patterns of high rates and the leveling off periods are essentially reproduced on all three curves, however, they are shifted temporally in terms of when they occur in the circadian cycle. For example, the initial increase in emission of carbon dioxide occurs in the September 25 data at around 10:00 A.M. and in the August 22 data it starts around 4:00 P.M., whereas on November 11 it is delayed until around 8:00 P.M. and is far less pronounced. The onset time of these high rate periods appears to occur later in the day as the total output of carbon dioxide becomes of lesser magnitude.

\section{Discussion of Results}

The infrared technique presented here may be utilized to examine behavior-physiological associated responses. Photoperiodic phasing is known to influence insect diapause (ADKisson, 1966) and the present study suggests that light phasing in addition alters respiration patterns when applied at certain stages of development. In the current literature, many studies have been reported which are aimed at examining the influence of various wavelengths of light and their effects on the biological clock and photoperiodic mechanisms in mammals. It is apparent, however, that more studies are needed which attempt to relate the overt behavior under field conditions to physiological alterations induced by a particular environmental stimulus. 
The demonstration of the stimulating influence of the BL insect attractant lamp on the respiration patterns indicates an endogenous response. The in-flight attraction for this type of light is obviously more than a simple behavioral effect. BüNNING (1967) discusses the possibility that phase shifting induced in several organisms by exposure to UV radiation may result from alterations in nucleic acid metabolism, although he also points out that attempts to confirm this hypothesis have not met with much success. From the data presented in Fig. 5 and in Table 2, it is suggested that the UV exposure is influencing rates of oxidative metabolism. The site of the action may be within the mitochondria which acts as a mediator in the light stimulus phase. If the BL light were influencing nucleic acid synthesis one would not expect the rapid return to a low carbon dioxide emission following an exposure (Table 2), to the UV source. An exposure to the BL lamp also appears to shift the pattern of diurnal release of carbon dioxide and, therefore, apparently alters the phasing of the biological process. This environmental stimulus does not, however, as shown by the data in Fig. 6 change the sequential pattern of the emission only the particular phasing in the circadian cycle and the total quantity of gas emitted.

\section{References}

AdKIsson, P. L. : Internal clocks and insect diapauses. Science 154, 234-241 (1966).

-, E. Vanderzant, D. L. Bull, and W. E. Allison: A wheat germ medium for rearing the pink boll Worm. J. econ. Entomol. 58, 759-762 (1960).

Agreit, I., in: Physiology of insecta, vol. I (M. Rockstein, ed.), p. 130. New York and London: Academic Press 1964.

BüNNTNG, E.: The physiological clock, p. 97. Berlin-Heidelberg-New York: Springer 1967.

Deay, H. O., J. R. Barrett, JR., and J. G. Hartstock: Field studies of flight response of Heliothis zea to electric light traps, including radiation characteristics of lamps used. Proc. North Cent. Branch Ent. Soc. Amer. 20, 1009-1019 (1965).

LeVENGOOD, W. C., and W. L. Wolfe: Optical method for monitoring the $\mathrm{pCO}_{2}$ level in physiological liquids. Proc. San Diego Symp. Biomed. Eng., vol. 4, LaJolla, Calif., p. 424-433 (1964).

Levy, R. I., and H. A. Schnemerman: Discontinuous respiration in insects. II. The direct measurement and significance of changes in tracheal gas composition during the respiratory cycle of silkworm pupae. J. Insect Physiol. 12, 83-104 (1966).

Mrller, P. L., in: Physiology of insecta, vol. III (M. Rockstein, ed.), p. 591594. New York and London: Academic Press 1964.

Slama, K.: Physiology of sawfly metamorphosis - I. Continuous respiration in diapausing prepupae and pupae. J. Insect Physiol. 5, 341-348 (1960).

\section{W. C. LEVENGOOD}

Institute of Science and Technology

The University of Michigan

P. O. Box 618

Ann Arbor, Michigan 48107 